Kansas State University Libraries

New Prairie Press

\title{
USING NONLINEAR GROWTH CURVES TO ESTIMATE HEAT STRESS IN PROCESSING FEEDLOT CATTLE
}

\author{
A. M. Parkhurst \\ T. L. Mader
}

Follow this and additional works at: https://newprairiepress.org/agstatconference

Part of the Agriculture Commons, and the Applied Statistics Commons

\section{(c) (1) $\Theta($}

This work is licensed under a Creative Commons Attribution-Noncommercial-No Derivative Works 4.0 License.

\section{Recommended Citation}

Parkhurst, A. M. and Mader, T. L. (2000). "USING NONLINEAR GROWTH CURVES TO ESTIMATE HEAT STRESS IN PROCESSING FEEDLOT CATTLE," Conference on Applied Statistics in Agriculture.

https://doi.org/10.4148/2475-7772.1244

This is brought to you for free and open access by the Conferences at New Prairie Press. It has been accepted for inclusion in Conference on Applied Statistics in Agriculture by an authorized administrator of New Prairie Press. For more information, please contact cads@k-state.edu. 


\title{
USING NONLINEAR GROWTH CURVES TO ESTIMATE HEAT STRESS IN PROCESSING FEEDLOT CATTLE
}

\author{
A. M. Parkhurst and T. L. Mader \\ Department of Biometry and Department of Animal Science \\ University of Nebraska-Lincoln
}

\begin{abstract}
Summertime heat waves cause excessive discomfort and, in extreme cases, death of feedlot cattle. During such emergencies, extension specialists are called upon for recommendations of management practices to minimize heat stress. Since moving cattle is believed to raise body temperature 1 degree, one recommendation is to move cattle before mid-day or reschedule to another day. More knowledge of body temperature dynamics could lead to more specific recommendations of how far cattle can be moved without stress. Several models are investigated - especially those involving exponential growth(challenge) and decay (recovery) such as the bi-exponential, single compartment and other models in pharmacokinetics. Data from feedlot trials can be "messy" and judgement calls involving starting and ending times, model parametrization, and statistical assumptions can influence the results. Analyzes from SAS: proc NLIN and checks on nonlinear assumptions are discussed.
\end{abstract}

\section{INTRODUCTION}

In the Great Plains and Western Corn-belt of the United States over 10 million head of cattle are being feed in feedlots at anyone time. Generally, cattle are processed (vaccinated, treated for parasites, provided a growth implant or an ear-tag for identification) within a few days of coming into the feedlot. In addition, most cattle are returned to the processing facilities to receive health care or be re-implanted with a growth promotant; plus, all cattle have to be moved or handled when they are shipped to the packing plant once they are finished. Because of these factors, a large number of cattle are being handled every week day of the year, regardless of the climatic conditions. Processing and handling of cattle requires an expenditure of energy most likely causing an elevation of body temperature, depending on the ambient conditions. The objective of this study was to evaluate effects of cattle movement in the feedlot and quantify body temperature of animals moved $~ 1200$ or 200 feet during an average summer day in August. These distances were selected to be within the range that feedlot cattle are normally moved to and from pens, although cattle can be moved greater or lesser distances.

The ultimate objectives of this study are to estimate and compare heat stress (challenge) and recovery time associated with each run length. In this paper, the focus is on estimation only. Heat stress is defined to be heat produced by moving steers a specified distance. It is measured as the temperature differential, the difference between the maximum and initial body temperature, TB. Additional parameters associated with heat production are time to reach 
maximum TB and rate of increase in body temperature. Another major objective is to estimate recovery time, RT, that is the time to return to initial $\mathrm{TB}$. The problem with this definition is the steer's TB seldom returns to the initial value. Alternatively, two parameters which could provide information about RT are the rate of heat dissipation and possibly a lower asymptote.

\section{METHODS}

Four head of cattle were assigned two to a pen. The pens were identical in size and bunk space. On day 1, cattle were weighed and tympanic data-loggers were placed in the left ear to record body temperature at 2-minute intervals. On days 2 and 3 (period 1), cattle in one pen were moved approximately 200 feet to the working facilities and returned (200 feet) back to their pen (treatment 1). Directly after returning cattle to that pen, cattle in the second pen were moved approximately 1200 feet, through working facilities, and then returned (1200 feet) back to their pen (treatment 2). Cattle were not handled on days 4 and 5. On days 6 and 7 (period 2) cattle in the two pens were assigned the opposite treatment and moved accordingly. Cattle were moved through facilities between 9 and $10 \mathrm{am}$.

\section{OBSTACLES TO ANALYSIS}

Some obstacles to analyzing these data are the treatment duration, recording frequency, messy data and variable conditions. The length of the treatment effect is difficult to assess. The time the treatment started and ended was recorded. Thus, the beginning of the treatment effect is defined to be the time the run started. But, the end of the treatment effect is more difficult to determine, since the effect of the treatment continues after the application stops. The TB continues to rise after the handling stops. One approach is to record the time it takes to return to the initial TB. But, as was previously mentioned, TB seldom returns to the initial TB. Another alternative is to plot the data and identify a lower asymptote. The end time of the asymptote could then represent the end of the treatment effect. Figure 1 shows potential asymptotes at 11/2, $2 \frac{1}{4}$, and $3 \frac{1}{2}$ hours.

Another issue is the frequency at which data is recorded. The data loggers were set to record TB every 2 minutes for 7 days. It is important to keep in mind taking readings at two minute intervals may miss the peak TB, particularly for the short distance treatment. Also changes in heat production may be difficult to distinguish from other fluctuations in the TB. The signal-tonoise ratio may obscure important characteristics of the data. Thus, the time scale is an important issue.

Messy data is another obstacle that needs attention. As with many field experiments, there are missing observations and unexplained spikes. Thermistor malfunction is always a possibility and the data needs to be "cleaned" or discarded when a malfunction is identified.

Finally, variable conditions raise other problems. It is impossible to control the ambient temperature, initial body temperature and distractions after the animals are moved. The ambient temperature is recorded but not controlled. It could be an unusually hot or cool day. Thus, the influence of climate is confounded with the treatment effect. Moreover, initial TB is not 
controlled. It varies from run to run. The animal may be on a temperature incline or decline when the treatment begins. How this situation influences the treatment effects is another unknown. Examples of uncontrolled distractions that may cause changes in TB are social interactions and body functions such as urination and defecation. Moreover, drinking and eating was not restricted.

\section{MODEL SELECTION}

Despite the complexity of processes involved in heat stress, temperature profiles produced by moving steers have similar patterns that can be described by mathematical models. There are many models that describe patterns of growth (heat challenge) and decline (recovery). Some models are purely empirical while others are mechanistic. Our main objective is to find a predictive model. To that end only the long run treatment was explored, for each of four steers with two replications Identifying a model that provides insight to the functioning of the biological process and generates testable hypotheses is an added incentive.

\section{4a. Normal and Lognormal Models}

A line plot provides a visual description of the data and highlights properties of the curve. Figure 1 shows we are looking for an empirical model that produces a questionably asymmetric concave curve with the possibility of one or more inflection points. We begin our list of candidate models with the ever-popular normal (Gaussian) distribution, Figure 2. Although the normal model has a maxima and two inflection points, it is symmetric and proc NLIN (SAS, 1999) failed to converge. Thus, a transformation to the lognormal model was necessary. This model proved plausible, Figure 3. Proc NLIN converged smoothly in 11 iterations. The MSE and standard errors are small. The $95 \%$ confidence intervals, for all but the first parameter, exclude zero in a plausible direction. The first parameter is not significantly different from zero, which is reasonable since it represents the initial increase in temperature. The correlations among parameters are reasonable, Table 1, and if we over look the pattern of hysteresis in the residuals, Figure 4, the lognormal can be added to our list of plausible models.

\section{4b. Exponential and Asymptotic Models}

The next two models address the question: Is the rate of change in body temperature exponential? The simplest mechanism of chemical kinetics is a monomolecular reaction in which a substance A is converted into a substance B. The Law of Mass Action says the rate of chemical reaction is proportional to the product of concentrations of the reactants. Here there is only one reactant and one forward velocity of reaction, since there is no back-reaction from $\mathrm{B}$ to $\mathrm{A}$. The rate of change can be written in terms of $\mathrm{A}$ or $\mathrm{B}$. When the rate of loss of $\mathrm{A}$ is assumed to be proportional to $\mathrm{A}$,

$$
\frac{\delta A}{\delta t}=-\kappa A
$$

the solution is the unbounded exponential decay model.

$$
A=A_{0} e^{-\kappa t} \quad \text { where } A_{0} \text { is initial amount of } A
$$


When $\mathrm{B}$, the rate of production is assumed to be proportional to the amount of $\mathrm{A}$ remaining

$$
\frac{\delta B}{\delta t}=-\kappa\left(A_{0}-B\right)
$$

the solution is the bounded exponential or asymptotic regression model, where $\mathrm{A}_{0}$ is the sum of the two concentrations, $\mathrm{A}$ and $\mathrm{B}$.

$$
B=A_{n}\left(1-e^{-x l}\right)
$$

The unbounded exponential model is used separately for the challenge and recovery and in combination for the bi-exponential. Although the bi-exponential curve, is asymmetric with a maximum, it severely under-estimates the starting value, $Y_{0}$ and the maximum, Figure 5. Lack of a bound and inflection point appears to be an issue, especially when modeling the heat challenge, Figure 6. Thus, the bi-exponential was crossed off the list of plausible models.

The results of the bounded exponential model, asymptotic regression, fit separately for both challenge and recovery are given in Figure 7 and for the bi-asymptotic model in Figure 8. Once again, the bi-asymptotic curve is asymmetric with a maximum; but, it severely under-estimates the starting value, $\mathrm{Y}_{0}$, and under-estimates the maximum. The presence of a bound produces a better fit for challenge; but, the lack of an inflection point still appears to be an issue. Thus, the bi-asymptotic model was also crossed off the list of plausible models.

\section{4c. Gunary and Extended Freundlich Models}

Other mechanistic considerations are based on modeling ion adsorption (Sibbesen, 1981). The assumption is the mobility of native ions and immobilization of added labile ions are controlled by adsorption reactions. The parameters may be interpreted as adsorption capacity and affinity properties of adsorbent for the adsorbate. This concept is used in agriculture to characterize soils for phosphate availability and suitability for application of fertilizers. In environmental studies, this concept is used to predict the potential for leaching into ground and surface water. A similar idea is used in enzyme kinetics and protein binding. It results in the rectangular hyperbolic model, also known as the Michaelis-Menten or emax model. The two models studied here are the Gunary, and the extended Freundlich.

The Gunary model is a modification of the Michaelis-Menten or Langmiur model. Gunary (1970) added a square root-term to the denominator to get a better fit.

$$
Y=\frac{X}{\alpha+\beta X+\gamma \sqrt{X}}
$$


The model can have a maximum or minimum at $X=4\left(\frac{\alpha}{\gamma}\right)^{2}$, and a pair of inflection points. Figure 9 and Table 2 show how well the model fits the data. The MSE and SE's are small. The CI's are small, exclude zero and are otherwise reasonable. The hysteresis in the residuals, Figure 10, shows the model fits different sections of the curve better than others. The model improves as the record length decreases from 204 to 86 minutes, Figure 9. The MSE decreases and the CI's become smaller (not shown). The model is plausible. The major difficulty is the parameters are difficult to interpret. For instance, $\alpha$, the reciprocal of the initial value may be zero.

The extended Freundlich is a modification of the Freundlich or power-growth model. The assumption is growth of $\mathrm{Y}$ is proportional to the power of $\mathrm{X}, \frac{\delta Y}{\delta X}=\phi X^{\gamma-1}$

The solution to this differential equation is $Y=\alpha X^{\beta X^{-\gamma}}$

The extended Freundlich has a pair of inflection points and a maximum or minium at

$$
X=e^{\frac{1}{\gamma}} \text {. If } \alpha \text { or } \beta \text { is negative, a minimum is obtained. Figure } 11 \text { shows that although the }
$$

overall fit is close, $\mathrm{MSE}=0.009$, the maximum is underestimated and the infection points do not match well. Furthermore, the CI for $\alpha$ includes zero, which makes the model difficult to interpret, Table 3. Hence, the extended Freundlich model is crossed-off the list of plausible models.

\section{4d. Extended Logistic and Bi-Logistic Models}

Another conceptualization is based on ligand-receptor binding. How much ligand binds to receptors in animals or plants? Examples of ligands are insulin, antibodys, and hormones in blood or other body fluids. Receptors may be sites on cell surface where ligand adheres. Two popular methods for estimating the concentration of ligands or degree of binding are the RIA, radioimmunoassay which uses a radioactive label for the ligand and ELISA, enzyme-linked immunoassay which uses fluorescent labels (Brown \& Rothery, 1993). These tests are standard practice in the pharmaceutical industry for drug development, laboratory research and clinical trials. They are also used to calibrate unknown test samples and to measure autocatalytic growth. In these cases, the focus is on ligand concentration rather than receptor availability and the logistic model is commonly used.

The logistic model is another example of limited growth. However, in this situation, the relative growth is not constant and is proportional to the amount remaining.

$$
\frac{1}{Y} \frac{\delta Y}{\delta X}=\kappa(\alpha-Y)
$$


The solution to the ODE gives a symmetrical sigmoidal curve with a rate of change which rises to an inflection point then falls, while the relative rate of change decreases linearly to an asymptote.

$$
Y=\frac{\alpha}{1+\beta e^{-\kappa X}}
$$

The asymptote $\alpha$ is commonly called the carrying capacity or equilibrium level; $\beta$ is a scale factor equal to the ratio of the range and the minimum; while, $\kappa$ is the rate constant or the intrinistic relative growth rate when the effects of limiting factors are negligible. The results of the logistic applied to challenge and recovery are given in Figure 12. Visually the plots show excellent fits, with small MSE and reasonable parameter estimates. The next step is to combine these results.

Brain and Cousens (1989) suggest extending the logit model by introducing a peak into the curve. They add a linear function of $\mathrm{X}$ to the asymptote in the numerator and indicate the additional parameter, $\gamma$, represents the initial rate of increase in the response at low levels of $X$. The results associated with the fit, illustrated in Figure 13, give MSE $=0.012$. However, $\alpha$, the initial TB is noticeably underestimated. It's CI, [-0.63 to -0.36$]$, does not include zero and this model is crossed-off the list of plausible models.

$$
Y=\frac{\alpha+\gamma X}{1+\left(e^{\tau} X\right)^{c}}
$$

Another tactic is to assume challenge and recovery are independent effects and that their combination can be predicted by an additive model.

$$
Y=\frac{\alpha_{1}}{1+\beta_{1} e^{\kappa_{1} X}}+\frac{\alpha_{2}}{1+\beta_{2} e^{\kappa_{2} X}}
$$

The resulting bi-logistic model is capable of having a maximum and pair of inflection points. Figure 14 and Table 4 show how well the bi-logistic model fits the 204 minute data record. The $\mathrm{MSE}=0.004$ and SE's are reasonable. The CI's are adequate and exclude zero. The hysteresis in the residuals, Figure 15, is also tolerable.

\section{MODEL PARAMETERIZATIONS AND TRANSFORMATIONS}

Statistical properties of nonlinear models may differ greatly from one parameterization to another. The parameterization most often resulting from solving the ODE using the common boundary conditions is 


$$
Y=\frac{\alpha}{1+\beta e^{\kappa X}}
$$

Since $\beta$ can be interpreted as the range of the data, we'll call this parameterization the range model. Another parameterization, which we'll call the inflection point model, is formed by reexpressing $\beta$ in terms of the inflection point, $\tau$, and substituting in Eq 1 .

$$
\beta=e^{-\kappa \tau}
$$

The resulting equation for the inflection point model is

$$
Y=\frac{\alpha}{1+e^{\kappa(X-\tau)}}
$$

The rate of change is symmetric about the inflection point and reaches a maximum of $\frac{\kappa \alpha}{4}$ at $X=\tau$, and $Y=\alpha / 2$, half the carrying capacity.

In dose-response curve analysis, the model most commonly used is based on a power of X. The slope factor, c, corresponds to the slope of the logit-log plot. This model represents a transformation of $\mathrm{X}$ rather than a reparameterization.

$$
Y=\frac{\alpha}{1+\left(\frac{X}{\tau}\right)^{c}}
$$

All three of these models are examined to find how the standard errors differ, Table 5. The inflection points parameterization shows the SE of $\tau_{1}=0.329$ is much smaller than the SE of $\beta_{1}$ in the range parameterization; while the SE of $\tau_{2}=4.273$ is only slightly larger than the SE of $\beta_{2}$ and much smaller than the SE of $\tau_{2}$ in the power model. Hence, the inflection point parameterization is recommended for this type of data.

\section{MODEL ASSUMPTIONS}

In addition to the classic assumptions about residuals, two additional assumptions are needed for least squares estimation of parameters in nonlinear regression: planarity and uniformity of coordinates. There are several ways to assess how close a model-data set combination is to satisfying these asymptotic properties. Bates and Watts (1980) proposed relative measures for intrinsic and parameter-effects curvature.

Intrinsic curvature(IN) measures the relative curvature of the expectation surface at the point of convergence. In linear regression, the surface is a plane and IN always equals zero. For nonlinear regression, IN increases as the curvatures of the expectation surface increases, invalidating the estimation procedure. Large IN values indicate unacceptable deviation from the tangent plane assumption. It is difficult to remedy this problem. Reparameterization does not 
alter the expectation surface. All parameterizations of a specified model have the same IN for a given data set. The only way to correct the problem is to get more data, a new model or transformation, or both.

Parameter-effects curvature, PE, measures the lack of uniformity of the parameter lines on the tangent plane. In linear regression, parameter contours are parallel and equally-spaced when projected onto the tangent plane. The PE value of maximum relative curvature is obtained from a scaled version of the Hessian i.e., the second derivatives of the model with respect to each parameter. For the linear case, all second derivatives equal zero resulting in a PE of zero. For nonlinear regression, a higher PE indicates higher degree of departure from the assumption of parallelism. The good news is problems with lack of uniformity can often be remedied by appropriate choice of parameters. Thus, the goal of reparameterization is to find a set of parameters that makes $\mathrm{PE}$ as small as possible.

Bates and Watts (1988) compare the maximum curvature values, IN and PE, to the curvature of the $95 \%$ confidence disk. They calculate the deviation of the expectation surface from the tangent plane at a distance $1 / \operatorname{sqrt}\left(\mathrm{F}_{.05, \mathrm{p}, \mathrm{n}-\mathrm{p}}\right)$ from the convergence point as a percentage of the radius of the $95 \%$ confidence disk. If this percent deviation is less than $21 \%$, the curvatures are deemed acceptable. Alternatively, the maximum curvature can be standardized by multiplying by $\mathrm{F}_{.05, \mathrm{p}, \mathrm{n}-\mathrm{p}}$. When the standardized maximum curvature measure is less than or equal to 0.4 , the curvatures are acceptable. Table 6 gives the results of curvature for the bi-logistic model. The planarity assumption does not hold for the power model, since the scaled IN is 1.38. However, planarity does appear acceptable for both parameterizations, since IN is 0.06 . The maximum curvature associated with PE is acceptable only for the inflection point parameterization.

Many times violations of assumptions are due to the properties of a single parameter. $\mathrm{PE}$ measures the maximum curvature associated with all the parameters. Thus, it is often of interest to examine each parameter individually. For linear regression models, under classical assumptions about the error term, the least-squares estimates are unbiased and normally distributed with minimum variance. For nonlinear regression, these properties hold only asymptotically. For real data sets, the statistical properties depend on both the model and the finite sample size. Ratkowsky (1990) suggests examining the "close to linear" behavior of each parameter. The measures used in this paper are Box's approximate measure of bias (1972), percent excess variance based on Lowry and Morton's asymmetry measure (1983) and Hougaard's approximate measure of skewness (1985).

Values for Box's approximate bias and bias expressed as a percentage of the least squares estimate are given in Table 7 . Using the under $1 \%$ rule-of-thumb for absolute \%bias suggests $\beta_{1}$ in the range model and all but $\tau_{1}$ and $\mathrm{c}_{1}$ in the power model are troublesome. However, all parameters in the inflection point parameterization appear to have close-to-linear behavior.

Lowry and Morton's asymmetry measure can be expressed as percentage of excess variance. Ratkowsky (quoted in Seber and Wild -1989, p 188) gives reference values for the measure. 
Expressed as a percentage, values $<1 \%$ indicate behavior is very close to linear; values between $1 \%$ and $5 \%$ indicate increasing skewness in the distribution of the parameter; and for values $>$ $5 \%$, skewness is very apparent in simulation. Results are given in Table 8 . Again troublesome parameters appear in the range $\left(\beta_{1}\right)$ and power (all parameters) models. None of the parameters appear troublesome in the inflection pt parameterization.

Skewness measures departure from symmetry. Hougaard's approximate skewness works well when $\mathrm{n}>150$. (Note: This statistic is now an option in Proc NLIN, version 8). Ratkowsky (1983) gives reference values for skewness. They are: $\mid$ skewness $\mid<0.1$ indicates behavior is very close to linear; between 0.1 and 0.25 , reasonably close to linear; between 0.25 and 1 , apparent skewness; and greater than 1, considerable skewness. Hougaard's skewness values for each parameter are given in Table 9. The range and power models have parameters with considerable skewness, while the inflection pts model appears to have closer-to-linear behavior.

\section{RESULTS}

The bi-logistic model, with the inflection point parameterization, was analyzed using three asymptotes to estimate recovery time. Results dependent on choice of asymptote ( record length). Picking a shorter recovery time gives a tighter fit, Figure 16. As the record length decreases from 204 to 86 minutes, the MSE decreases from 0.004 to 0.001 . The maximum change in body temperature changes only slightly from 1.79 to 1.89 . The time to reach the maximum heat stress is also robust. It changes from 41.7 to 41.1 minutes. The rate of challenge changes from -0.1860 to $-0.1863 \mathrm{C} / \mathrm{min}$. The rate of recovery is more variable, ranging from -0.03 for $31 / 4$ hours to $-0.08 \mathrm{C} / \mathrm{min}$ for 1.5 hours..

\section{SUMMARY}

Eight models were studied. The change in body temperature does not appear to follow a normal (Gaussian) curve nor does it follow a combination of unbounded (bi-exponential) or bounded (bi-asymptotic) models, nor an extended Freundlich, nor an extended logistic model. However, other models are plausible, including the lognormal, Gunary, and bi-logistic. The authors judged the bi-logistic to be most useful. It provides information about the time and amount of maximum heat challenge and rate of increase in body temperature. Time to recovery is more elusive, since it depends on record length. Two parameterizations and a transformation of the bi-logistic were also studied using Bates and Watts measures of maximum curvature, Box's approximate percent bias, Lowry \& Morton's percent excess bias, and Hougaard's approximate skewness. The parameterization based on the inflection points met the above criteria and gave shorter confidence intervals. Thus, the authors recommend using the bi-logistic model with the inflection point parameterization, appropriate checks on residuals and checks on "close-to-linear" behavior to analyze data from handling trials. It is also important to increase the frequency of recording observations. Ideally, observations should be recorded more than every two minutes, especially when the treatments involve handling cattle for shorter distances. 


\section{ACKNOWLEDGEMENTS}

The authors wish to thank Drs. David Meeks and William Price for their stimulating comments and Yuli Xie for providing help with the analysis, figures and tables.

\section{REFERENCES}

Bates, D. M., and Watts, D.L. 1980, "Relative Curvature Measures of Nonlinearity" J.R.Statist. Soc. Ser.B 42:1-25

Bates \& Watts. 1988. Nonlinear Regression Analysis \& Its Applications. John Wiley \&Sons, New York

Box, M.J. 1972. “Bias in nonlinear estimation”,J.R.Statist. Soc. Ser.B 33:171-201

Brain, P. and R. Cousens. 1989. "An equation to describe dose responses where there is stimulation of growth at low doses", Weed Research 29:93-96

Brown, D. and P Rothery. 1993. Models in Biology John Wiley \&Sons, New York.

Gunary, D. 1970. "A new adsorption isotherm for phosphate in soil” J. Soil Science. 21:72-77

Hougaard, P., 1985. "The appropriateness of the asymptotic distribution in a nonlinear regression model", J.R.Stat. Soc. Ser.B 47:103-114

Lowry, R. and R. Morton, 1983. "An asymmetry measure for estimators in non-linear regression models", Proc. $44^{\text {th }}$ Session Int. Statist. Inst., Madrid, Contributed Papers 1:351-354

Rathowsky, D.A. 1983, Nonlinear Regression Modeling: A Unified Practical Approach.Marcel Dekker: New York and Basel.

Ratkowsky, D. 1990, Handbook of Nonlinear Regression Models. Marcel Dekker, New York and Basel.

SAS 1999. SAS/STAT User's Guide, Version 8, Online.Com, SAS Institute Inc., Cary, NC

Seber G, and C. Wild, 1989. Nonlinear Regression. John Wiley \&Sons, New York

Sibbesen, E. 1981."Some new equations to describe phosphate sorption by soils", J. Soil Science 32:67-74. 
Figure 1: Observed Body Temperature

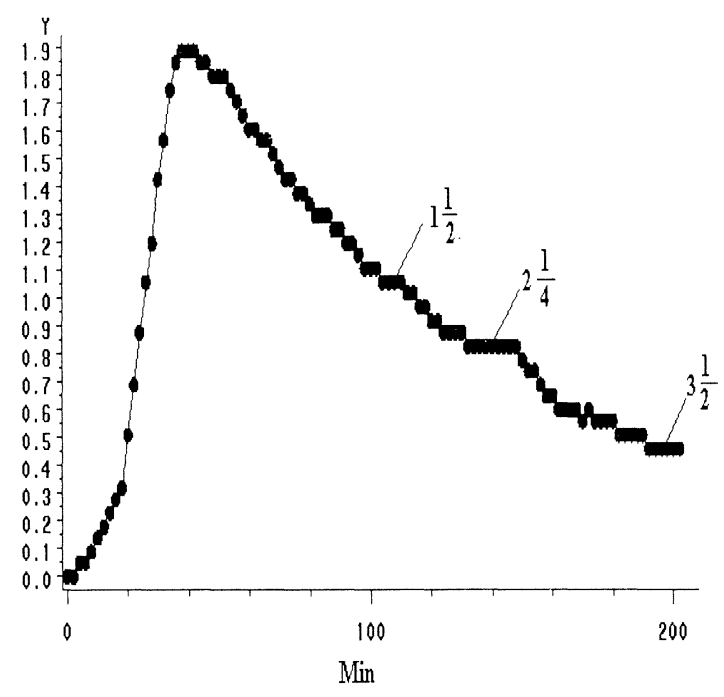

Figure 4: Normal Model

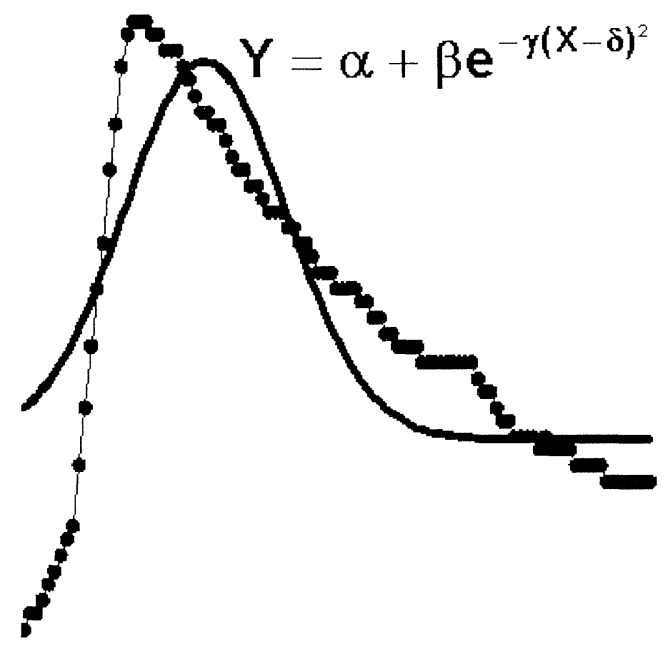

Figure 3: Lognormal Model (146min)

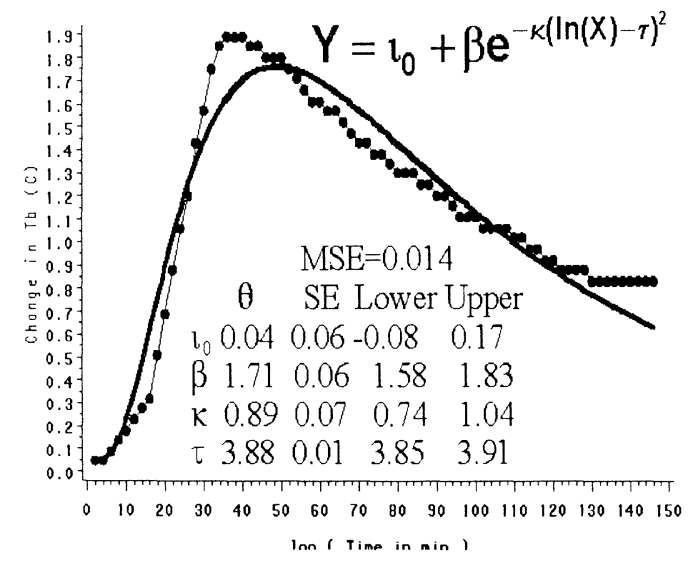

Figure 2: Studentized Residual

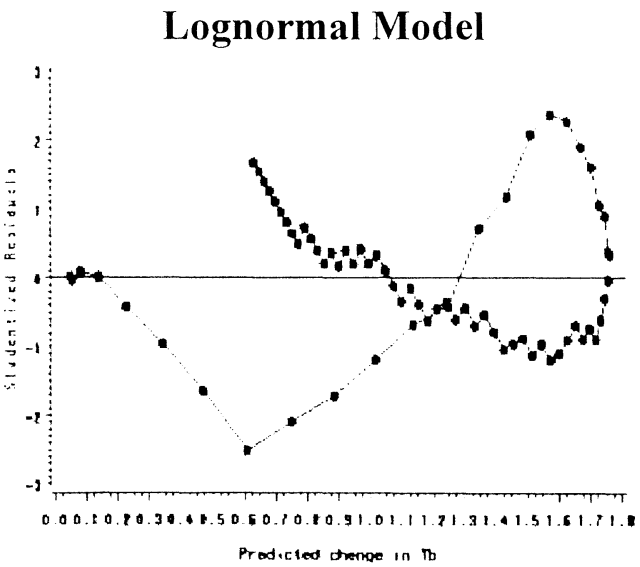


Figure 5: Unbounded Bi-exponential

Table 1: Lognormal Model Correlations

\begin{tabular}{|c|c|c|c|c|}
\hline & $\boldsymbol{l}_{0}$ & $\beta$ & $\kappa$ & $\tau$ \\
\hline $\boldsymbol{l}_{0}$ & 1 & -0.90 & 0.81 & 0.16 \\
\hline$\beta$ & & 1 & -0.60 & -0.18 \\
\hline$\kappa$ & & & 1 & 0.35 \\
\hline$\tau$ & & & & 1 \\
\hline
\end{tabular}

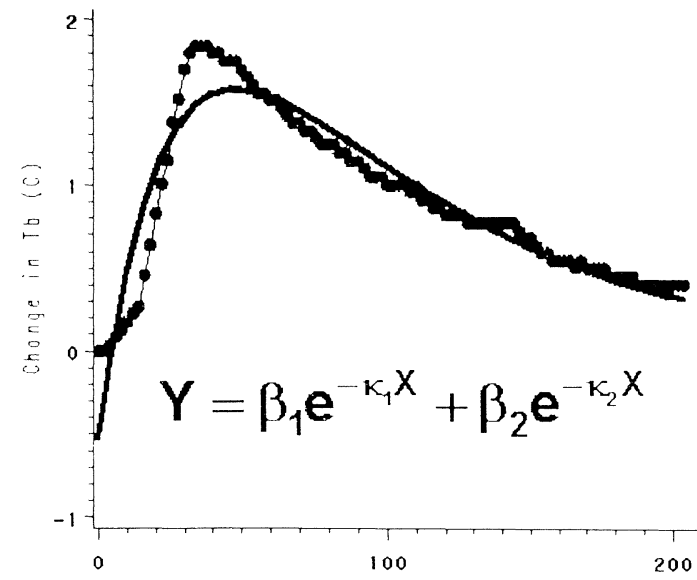

Figure 6: Unbounded Exponential $\quad Y=\beta e^{\kappa X}$

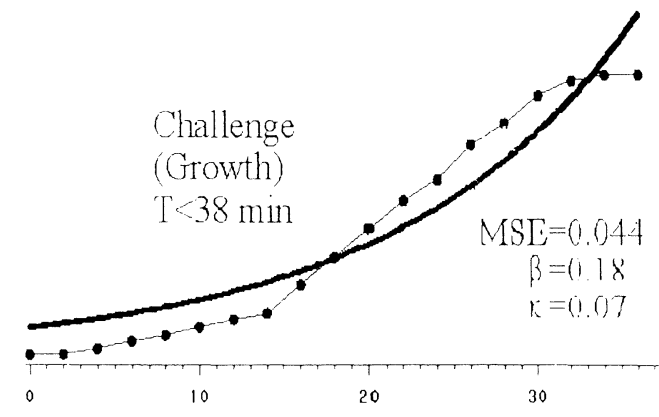

Figure 7: Bounded exponential Asymptotic regression

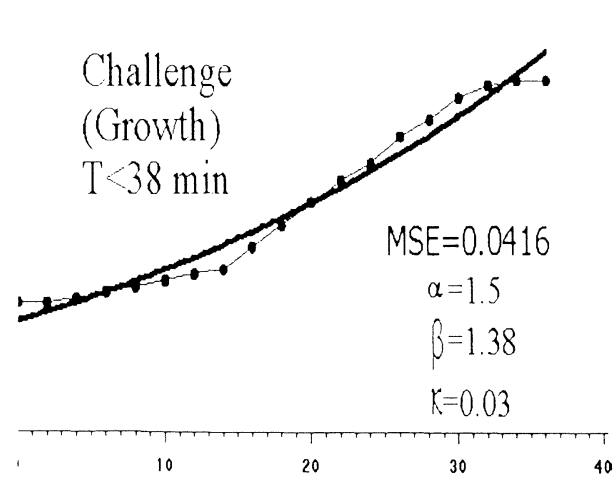

$\mathbf{Y}=\alpha-\beta \mathbf{e}^{-\kappa \mathbf{X}}$

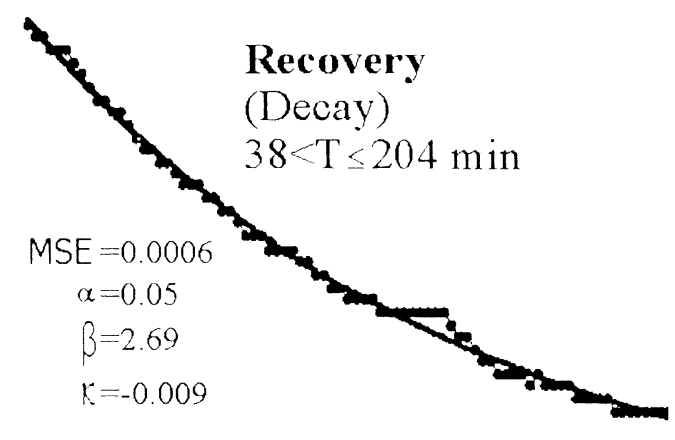


Figure 8: Bi-asymptotic Model Bounded Bi-exponential

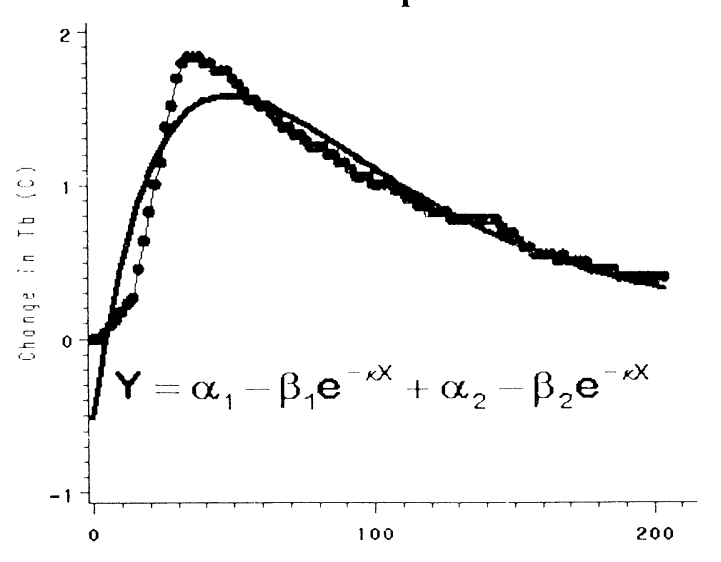

Figure 9: Gunary Model

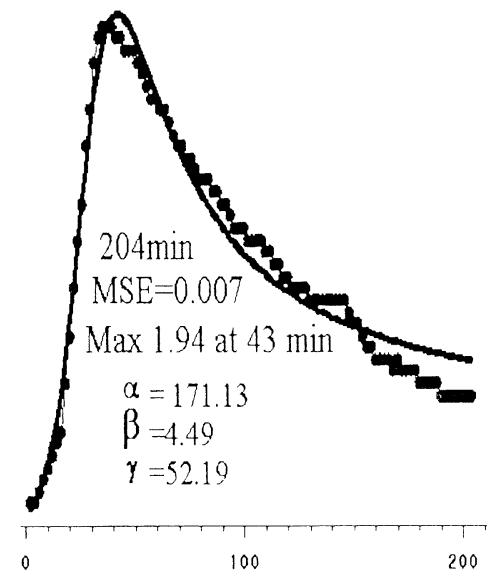

$\mathbf{Y}=\frac{\mathbf{X}}{\alpha+\beta \mathbf{X}+\gamma \sqrt{\mathbf{X}}}$

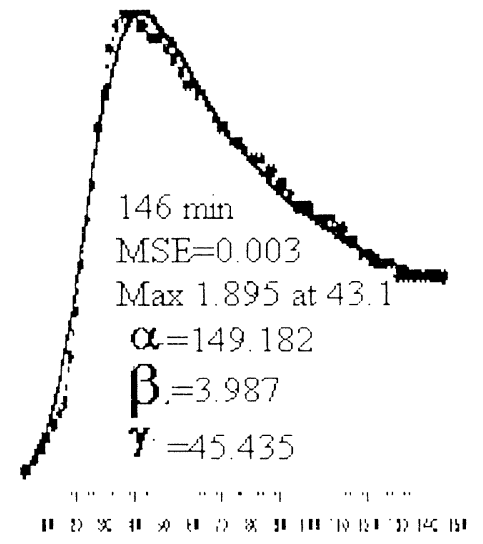

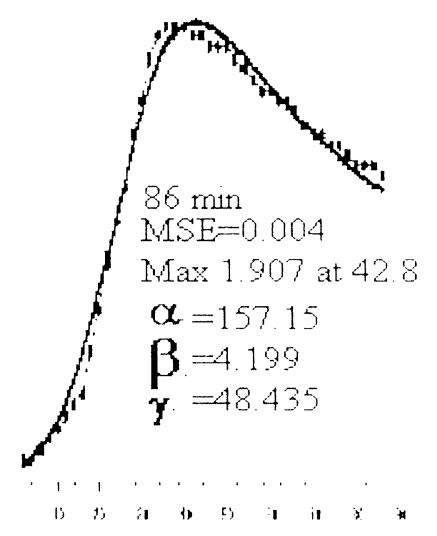

Figure 10: Studentized Residuals-Gunary

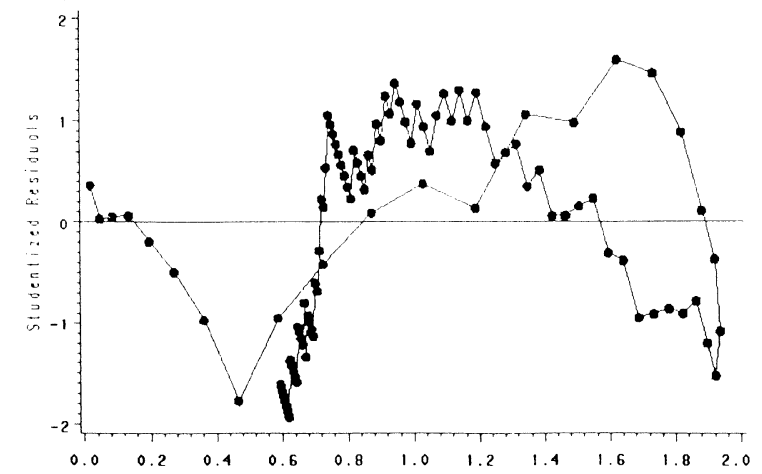

Table 2: Gunary Parameters (204 min)

\begin{tabular}{|c|c|c|c|c|}
\hline & $\theta$ & SE & Lower & Upper \\
\hline$\alpha$ & 171.13 & 5.39 & 160.43 & 181.83 \\
\hline$\beta$ & 4.49 & 0.10 & 4.2913 & 4.70 \\
\hline$\gamma$ & 52.19 & 1.50 & 49.22 & 55.17 \\
\hline
\end{tabular}


Figure 11: Extended Freundlich Model

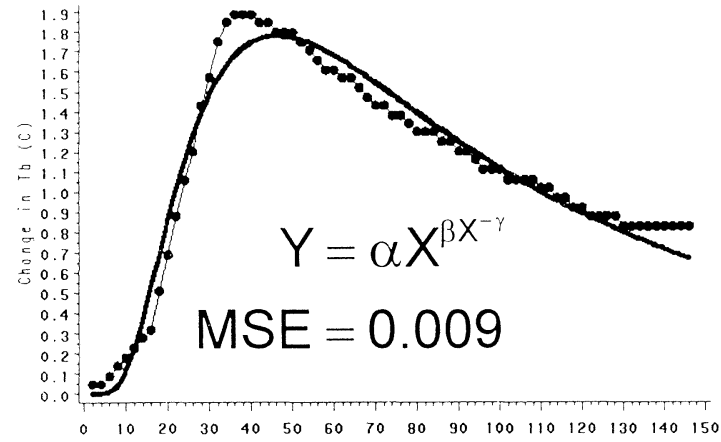

Figure 12:: Logistic Model

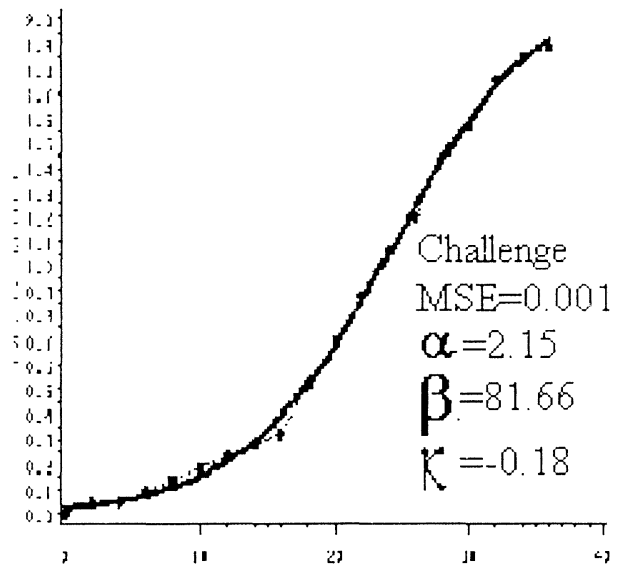

Table 3: Freundlich Parameters (146 min)

\begin{tabular}{|c|c|c|c|c|}
\hline & $\theta$ & SE & Lower & Upper \\
\hline$\alpha$ & 0.0000 & $0.1 \mathrm{E}-10$ & 0.000 & 0.000 \\
\hline$\beta$ & 18.7534 & 0.6755 & 17.406 & 20.100 \\
\hline$\gamma$ & $\begin{array}{l}\text { Covery } \\
\text { SE }=0.000 \\
\alpha=59 \\
\beta=21.08 \\
K=0.009\end{array}$ & $\begin{array}{r}\alpha \\
+\beta\end{array}$ & 0.25 & 0.262 \\
\hline \multicolumn{5}{|c|}{ 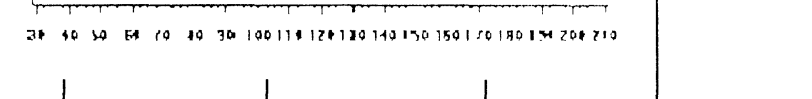 } \\
\hline
\end{tabular}

Figure 13: Extended Logistic Model

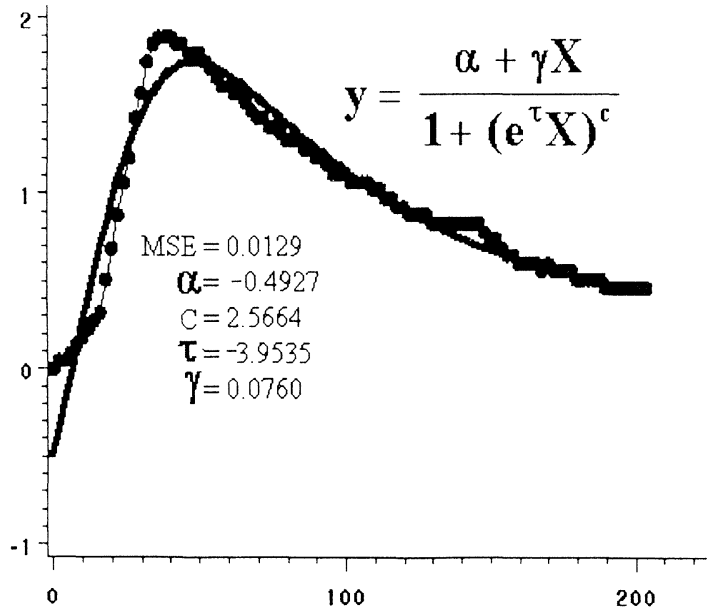


Figure 14: Bi-logistic Model (204 min)

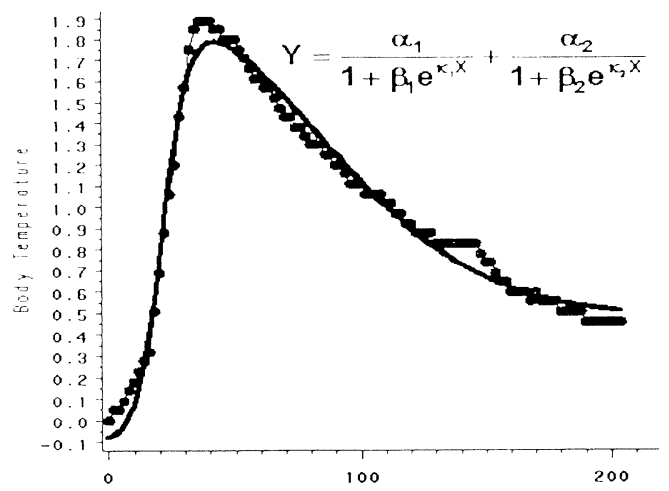

Figure 16: Bi-logistic for 3 record lengths
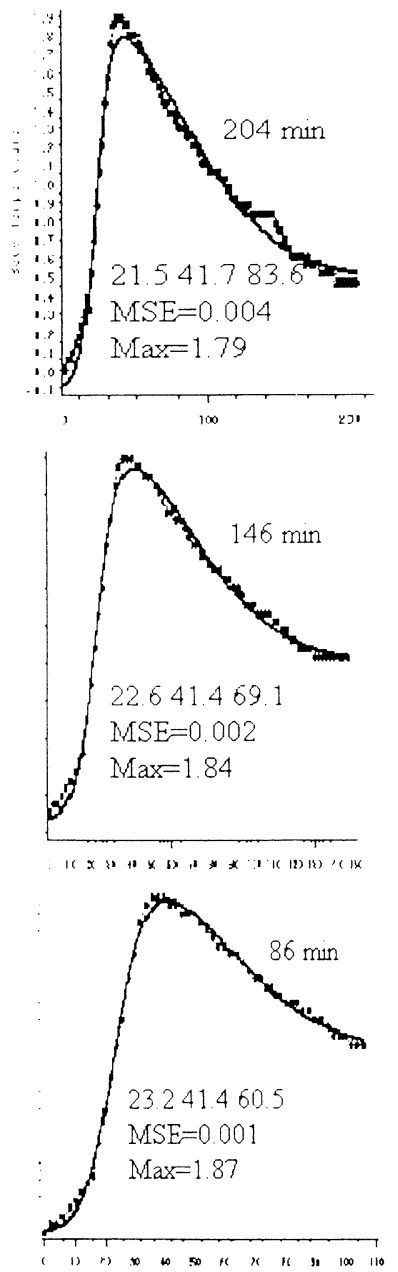

Figure 15: Studentized residuals Bi-logistic Model

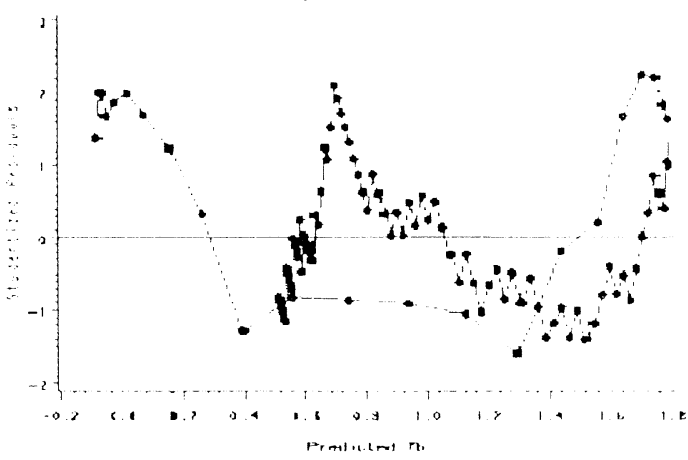

Table 4: Parameters for Bi-logistic

\begin{tabular}{|c|c|c|c|c|}
\hline & $\theta$ & SE & Lower & Upper \\
\hline$\alpha_{1}$ & 2.209 & 0.110 & 1.991 & 2.428 \\
\hline$\beta_{1}$ & 54.322 & 18.654 & 17.298 & 91.346 \\
\hline$\kappa_{1}$ & -0.186 & 0.015 & -0.216 & -0.156 \\
\hline$\alpha_{2}$ & -1.737 & 0.122 & -1.981 & -1.494 \\
\hline$\beta_{2}$ & 13.328 & 3.964 & 5.460 & 21.197 \\
\hline$\kappa_{2}$ & -0.030 & 0.002 & -0.035 & -0.026 \\
\hline
\end{tabular}

Table 5: SE for Parameterizations of Bi-logistic

\begin{tabular}{|c|c|c|c|}
\hline$\theta$ & Range & Inflection pts & Power \\
\hline$\alpha_{1}$ & 0.110 & 0.110 & 0.354 \\
\hline$\beta_{1}: \tau_{1}$ & 18.654 & 0.329 & 0.401 \\
\hline$\kappa_{1}: c_{1}$ & 0.015 & 0.015 & 0.483 \\
\hline$\alpha_{2}$ & 0.122 & 0.122 & 0.466 \\
\hline$\beta_{2}: \tau_{2}$ & 3.964 & 4.273 & 9.299 \\
\hline$\kappa_{2}: c_{2}$ & 0.002 & 0.002 & 0.363 \\
\hline
\end{tabular}

Table 6: Standardized Maximum Curvatures

\begin{tabular}{|l|l|l|l|}
\hline & Range & Inflection pts & Power \\
\hline IN & 0.06 & 0.06 & 1.36 \\
\hline PE & 0.939 & 0.364 & 13.205 \\
\hline
\end{tabular}


Table 7: Box's Bias and \%Bias

\begin{tabular}{|c|c|c|c|c|c|c|}
\hline Model & \multicolumn{2}{|c|}{ Standard } & \multicolumn{2}{c|}{ Inflection Points } & \multicolumn{2}{c|}{ Power } \\
\hline Parameter & Bias & $\%$ Bias & Bias & $\%$ Bias & Bias & $\%$ Bias \\
\hline$\alpha_{1}$ & 0.002 & 0.100 & 0.002 & 0.100 & 0.060 & 2.427 \\
\hline$\beta_{1}: \tau_{1}$ & 1.513 & 2.313 & -0.004 & -0.017 & -0.127 & -0.523 \\
\hline$\kappa_{1}: c_{1}$ & -0.000 & 0.065 & -0.000 & 0.065 & -0.001 & 0.032 \\
\hline$\alpha_{2}$ & -0.002 & 0.140 & -0.002 & 0.140 & -0.046 & 1.935 \\
\hline$\beta_{2}: \tau_{2}$ & -0.077 & -0.560 & -0.079 & -0.093 & -1.710 & -1.994 \\
\hline$\kappa_{2}: c_{2}$ & 0.000 & -0.120 & 0.000 & -0.120 & -0.085 & 4.340 \\
\hline
\end{tabular}

Table 8: Lowry and Morton's \%Excess Variance

\begin{tabular}{|c|c|c|c|}
\hline Parameter & Range & Inflection points & Power \\
\hline$\alpha_{1}$ & 0.208 & 0.207 & 50.507 \\
\hline$\beta_{1}: \tau_{1}$ & 1.271 & 0.106 & 49.190 \\
\hline$\kappa_{1}: c_{1}$ & 0.168 & 0.168 & 35.510 \\
\hline$\alpha_{2}$ & 0.189 & 0.188 & 47.227 \\
\hline$\beta_{2}: \tau_{2}$ & 0.150 & 0.185 & 46.574 \\
\hline$\kappa_{2}: c_{2}$ & 0.103 & 0.103 & 56.855 \\
\hline
\end{tabular}

Table 9: Hougaard's Approximate Skewness

\begin{tabular}{|c|c|c|c|}
\hline Parameter & Range & Inflection points & Power \\
\hline$\alpha_{1}$ & 0.282 & 0.284 & 1.030 \\
\hline$\beta_{1}: \tau_{1}$ & 1.241 & -0.130 & -0.418 \\
\hline$\kappa_{1}: c_{1}$ & -0.231 & -0.230 & 0.010 \\
\hline$\alpha_{2}$ & -0.182 & -0.184 & -0.508 \\
\hline$\beta_{2}: \tau_{2}$ & 1.401 & -0.197 & -1.230 \\
\hline$\kappa_{2}: c_{2}$ & -0.602 & -0.602 & -1.439 \\
\hline
\end{tabular}

